\title{
A Self-Optimization of the Dynamic Resource Management Based on the Cognitive Radio
}

\author{
Jamal Raiyn \\ Qasemi Research Center, Al-Qasemi Academic College of Education, Baka El-Gharbia, Israel. \\ Email: rayan@qsm.ac.il
}

Received August 21 ${ }^{\text {st }}$,2010; revised November 16 $6^{\text {th }}$, 2010; accepted November $19^{\text {th }}, 2010$.

\begin{abstract}
This paper describes a novel self-optimized approach for resource management based on the cognitive radio in the cellular networks. The cognitive radio techniques offer several features like autonomy, sensing and negotiation. The use of cognitive radio approach gives greater autonomy to the base stations in the cellular networks. This autonomy allows an increase in flexibility to deal with new situations in the traffic load. The negotiation strategy is used to avoid conflicts in the resource allocation. The goal of the cognitive radio scheme is to achieve a high degree of resource usage and a low rate of call blocking in the cellular systems.
\end{abstract}

Keywords: Cognitive Radio, Radio Spectrum Sensing, Social Agent

\section{Introduction}

Various resource assignment strategies [1-5] are proposed to increase the efficiently utilization of the radio spectrum [6] by reducing the call blocking rate [7] in the cellular systems. The call blocking probability is often measured in terms of two blocking probabilities, the arriving call blocking probability, and the handoff blocking probability. Analyses and studies in $[8,9,10]$ show that the call blocking Probability is caused by two parameters, co-channel allocation interference and allocation delay. The co-channel interference, when not minimized, decreases the ratio of carrier to interference power at the periphery of the cells, causing diminished system capacity, more handoff [11], and more dropped calls. However, most of the solutions proposed have an entirely reactive approach: the response to a series of events follows an algorithm that is designed to react to a specific situation, this limits their efficiency. The delay and the interference in cellular systems are considered as the main reason limiting the capacity and increasing the handoff call blocking probability. We note that in some application fields like real-time communication it is necessary to ensure a seamless and lossless handoff. In order to overcome the disadvantage of proposed methods, and to meet the new generation mobile communication service requirements, we introduce the cognitive radio approach. Cognitive radio is a new and novel way of thinking about wireless communications. Using the cognitive radio in the cellular systems will lead to a revolution in wireless communication with significant impacts on technology as well as regulation of spectrum usage to overcome existing lacks. The cognitive radio is a self-aware communication system that efficiently uses spectrum in an intelligent way. Spectrum sensing defined as the task of finding spectrum holes by sensing the radio spectrum in the local neighborhood of the cognitive radio receiver in an unsupervised manner $[12,13]$. The term "spectrum holes" stands for those sub-bands of the radio spectrum that is underutilized at a particular instance of time and specific geographic location. The task of spectrum sensing involves the following subtasks, detection of spectrum holes, spectral resolution of each spectrum holes, estimates of the spatial directions of incoming interference, and signal classification. After sensing, is to find a good transmission strategy that is aware of its surrounding environment to adapt their transmission parameters to the environment and the interference situation. It autonomously coordinates the usage of spectrum in identifying unused radio spectrum based on observing spectrum usage. Cognitive radios provide besides cognition in radio resource management also cognition in services and applications. The novelty of the cognitive radio system is expressed in the self-optimization tools. A short term forecast scheme has been proposed to optimize the cognitive radio rules in the system. In our forecast model, the forecast is done in real time. The forecast model is 
focus on the traffic systematically based on the mobile user's properties and on the information about the environment, we shall use, mathematical models to forecast, in real time, and the short time behavior of the mobile users. We have developed a forecast algorithm that takes as input the real-time observations. Based on the realtime forecast, the cognitive radio system improves the adaptability and management of the computational resource in the cellular systems. We have developed a forecast algorithm that takes as input the on-line measurements of RSS and the link quality to forecast the channel behavior. The proposed forecast scheme is based on the exponential moving average. Exponential moving average $[14,15]$ is a very popular scheme to produce a smoothed Time Series. Whereas in Moving Averages the past observations are weighted equally, Exponential Smoothing assigns exponentially decreasing weights as the observation get older. In other words, recent observations are given relatively more weight in forecasting than the older observations. The given weight determines how responsive a forecast is to sudden jumps and drops. It is the percentage weight given to the prior period, and the remainder is distributed to the other historical periods. The weight alpha is used in all exponential smoothing methods. Various theoretical and empirical arguments for selecting an appropriate smoothing parameter have been discussed. The paper is organized as follows: Section 2 introduces the system model and the social agent strategies. Section 3 describes the comparison between the sensors data and information bases mobile services. Section 4 and Section 5 discuss and analyze the simulation results of the optimized smoothed parameters and conclude the paper.

\section{Cognitive Resource Management}

A cellular network consists of an array of cells. We partition the cellular network to clusters. For each cell we set a social agent, as depicted in Figure 1. By using radio cognitive approach we aim to achieve an optimal network capacity, minimizing interference to other signals and to reduce messages complexity and channel acquisition delay that are considered the main reasons to block the new calls. A radio cognitive approach may be able to sense the current spectral environment, and have information of past transmitted and received packets along with the power, bandwidth, and modulation. By considering all this, it makes better decisions about how to best optimize for some overall goal. Under heavy traffic load, and if a vacant channel is not found, the social agent then tries to obtain an exclusive channel by optimization of channel distribution.

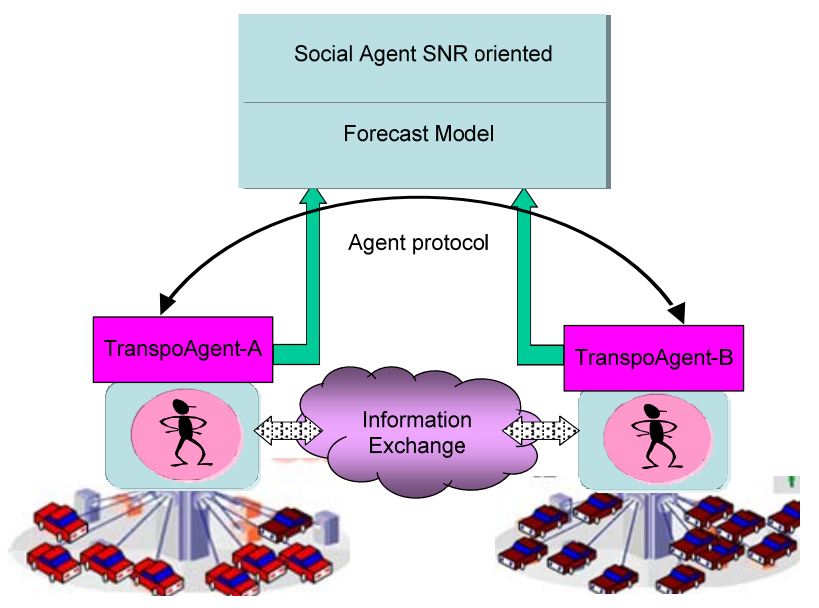

Figure 1. System model.

\subsection{Active Sense Environment}

Numerous spectrum sensing techniques have been proposed [13]. However one of the most important components of the cognitive radio is the ability to measure, sense, update, learn, and be aware of the following parameters related to the radio channel characteristics: propagation model, traffic model and amount of the information. This represents the maximum amount of information that can be conveyed through a communications channel. From an information theoretic perspective, a communications channel is responsible for passing data between two points, and will likely add some sort of noise to be original signal. Spectrum sensing is a major task in the cognitive radio communications, as it enables the cognitive radio to adapt its environment by detecting spectrum holes. In this paper we introduce a new and effective way to detect the availability of some portions of spectrum is to detect the mobile users that are receiving data within the range of a cognitive radio. The social agent uses a modern strategy that is based on the cellular phone service. Based on the information collection the social agent can update it local information and forecast which cells are with the heavy traffic load in non uniform conditional time. After the sensing, the next step for social agent is to find a good transmission strategy.

\subsection{Social Agent Decision Strategy}

In this phase the social agent deals with handover request. The social agent's decision about the handover process is focused on quality of service requirements (e.g. signal power, signal-to-noise ratio and delay). The signalto-noise ratio (SNR or S/R) defined as the ratio of a signal power to the noise power corrupting the signal. The social agent estimates the SNR and then determines to carry out the handover or not. Furthermore the agent collects information about the adjacent cells. Based on of 
the collected information from the adjacent cells, the agent determines the next handover cell. The handover based on SNR can be divided into two main categories:

1) The first scenario is based on received SNR from the base station only. This method decides handoff when the SNR from current station is smaller than another station. This kind of method is simple but will take place repeated or unnecessary handoff.

2) The second scenario is based on relative SNR with threshold. In this approach, handoff is initialed when the average SNR falls below a certain threshold value. This method can avoid unnecessary handoff when the current station signal is still satisfactory.

\subsection{Social Agent Negotiation Strategy}

Compared to the traditional negotiation strategy that offers high messages complexity, we are interested in applications where negotiation between social agents serves to solve the resource allocation problem in cellular systems. Furthermore we anticipate concern the feasibility of reaching an allocation of resources that is optimal from a social point of view. Social agents often need to interact in order to improve their performance. One type of interaction that is gaining an increasing interest is dynamic negotiation. The goal of negotiation is the maximization of the utility of a future decision. In distributed dynamic environment, each cell has an objective that specifies its intention to acquire a free channel for call establishment. That objective should be achieved in a certain amount of time, specified by a deadline. Negotiation stops when this deadline is reached.

\subsection{Social Agent Reasoning Strategy}

The cognitive radio approach draws its reasoning based on employing the forecast scheme. The forecast scheme uses the exponential moving average. The exponential moving average is expressed as follows:

$$
t t(t+1, k)=\alpha * t t^{M}(t, k)+(1-\alpha) * t t^{H}(t, k)
$$

where $0<\alpha \leq 1 . t t^{M}(t, k)$ is actual information at time $t$ in the section $k, t t^{H}(t, k)$ is historical information. The exponential moving average gives more weight to the latest (most recent) observation data and gives less weight to the older, more historical data. An exponential moving average actually reacts faster to recent observation changes than a simple moving average. The idea behind the exponential moving average is that it provides stronger and earlier trend detection. Alpha determines how responsive a forecast is to sudden jumps and drops. It is the percentage weight given to the prior period, and the remainder is distributed to the other historical periods.

\subsection{Social Agent Beliefs}

The new call in cell is blocked when there are no more free channels in the cell or the QoS requested cannot be provided as the SIR is under a given threshold $\mathrm{SIR}^{\mathrm{tgt}}$. By computing the call blocking probability in handover process, we consider four social agent decision scenarios that are described below.

1) Probability (Approve, $S N R>S N R^{\text {tgt }}$ )

2) Probability (Reject, $S N R<S N R^{\text {tgt }}$ )

3) Probability (Approve, SNR $<\mathrm{SNR}^{\mathrm{tgt}}$ )

4) Probability (Reject, $S N R>S N R^{\text {tgt }}$ )

From social agent view point, there are two kinds of decision. Good decision, $\mathrm{B}(1)$ and bad decision, $\mathrm{B}(0)$. The mentioned four scenarios can be expressed in binary information as follow: $\mathrm{B}(1,1), \mathrm{B}(0,0), \mathrm{B}(1,0), \mathrm{B}(0,1)$.

\subsection{Non Condition Traffic Identification}

The cognitive radio system is characterized that senses and is aware of its operational environment and can dynamically and autonomously adjust its radio operating parameters accordingly. A radio frequency transmitter/ receiver that is designed to intelligently detect whether a particular segment of the radio spectrum is currently in use, and to jump into (and out of, as necessary) the temporarily-unused spectrum very rapidly, without interfering with the transmissions of other authorized users”. In order to deal with real-time mobile communications the social agent has to change its transmitter parameters based on the interaction with the environment in which it operates." First of all, the social agent forecasts the traffic load in real-time. The social agent updates its historical information. The social agent makes a forecast for real-time based on the historical information and the real-time information.

$$
t t(t+1, k)=t t^{H}(t+1, k)+\gamma *\left[t t^{H}(t, k)-t t^{M}(t, k)\right]
$$

where $0<\gamma<1$. Gamma is an optimized parameter that gives higher weight for real-time information.

\subsection{Interference Area Identification}

In a mobile system, since the mobile stations can move between cells, the number of mobile stations within a cell at a given time can never be known exactly in advance. However, we can estimate roughly the location of the mobile stations. To avoid the call blocking by handover, it is important to identify the interference area. The interference area can be considered as the node of decision to hand over to a new cell. Denote $P_{t}$ as the transmitter power of the base station, $G$ the antenna gain, $d$ the distance between the transmitter and receiver and $N_{0}$ as the thermal noise power. Generally, then, the received signal-to-noise ratio (SNR) at the $k$-th user in cell-1 is given 


$$
\begin{aligned}
& \operatorname{SNR}_{1\left(d_{1}\right)}^{1}=\frac{P_{t} G\left(d_{1}\right)}{N_{0}}, S N R_{2\left(d_{2}\right)}^{1}=\frac{P_{t} G\left(d_{2}\right)}{N_{0}}, \cdots, \\
& \operatorname{SNR}_{j\left(d_{k}\right)}^{1}=\frac{P_{t} G\left(d_{k}\right)}{N_{0}}
\end{aligned}
$$

for $j=1, \cdots, k$. The signal-to-noise ratio (SNR or $S / R$ ) $S N R_{k}$ defined as the ratio of a signal power to the noise power corrupting the signal. The social agent can forecast the average blocking probability of user $k$ being served by the base station based on calculation of the interference area for cell $i$, which is greater than a given target value for cell $i, S N R_{i}^{\text {tgt }}$. This blocking probability is:

$$
P_{b}=\frac{1}{n} \sum_{k=1}^{n} \delta_{k}
$$

where $\delta_{k}=\left\{\begin{array}{ll}1 & \left(S N R_{k}-S N R_{i}^{t g t}\right)<0 \\ 0 & \text { Otherwise }\end{array}\right.$.

\section{Local Information Updating}

The social agent updates it local information based on the travel data collection. There are two ways for the travel data collections; the first is the traditional strategy that is based on the sensors and cameras, the second is the modern strategy that is based on the cellular systems. In the last time (second half of $20^{\text {th }}$ century) the phenomenon of traffic congestion has become predominant due to the rapid increase in the number of vehicles. Traffic congestion appears when too many vehicles attempt to use a common transportation infrastructure with limited capacity. General, the traffic information collected based on sensors or cellular systems. Conceptually, traffic information may fall into one of the three categories as follows; Historical information, real-time information, and Predictive information. The historical data is a collection of past observations of the system. Historical data describe the traffic states of a transportation system during previous time periods. It is mainly used to classify daily graphs or special events. Real-time information is most up-to-date and can be calculated, e.g., by on-line simulations. Predictive information, like traffic forecasts, can help to change the travel behavior of road users by providing information about the future state of the network. Figure 2 illustrates the travel data information that has been collection by the sensors and cellular mobile. The travel observation is collected in interval of 2.5 minute.

\section{Optimized Smoothed Factors}

To achieve a robust forecast model, it is needed to collect accurate travel information; firstly it is necessary to consider the optimization of the resource allocation in the cellular systems. The optimization of the resource allocation in the cellular system consider various issues like unnecessary repeated handoff, radio spectrum coverage, call blocking probabilities, delay and interference. Our analysis has been shown that the mentioned issues are strongly influence the quality of the collected travel flow information. In this case we use the collected information based on the cellular mobile services to detect the lack of the radio spectrum coverage and to specify the handoff blocking rate. To find the optimal value for the optimized smoothed parameter alpha we use the least squares fitting methods. The least squares fitting methods has been given in (4).

$$
\begin{aligned}
& \underset{\alpha^{\prime} \gamma}{\operatorname{mininize}}(J) \text { : } \\
& \text { s.t. } \quad J=\sum_{\text {zone time }} \sum_{\mathrm{t}}\left(\tilde{\mathrm{t}}_{\mathrm{t}}-t \mathrm{tt}_{\mathrm{t}}^{M}\right)^{2}
\end{aligned}
$$

Figure 3 illustrates the value of the optimized smoothing parameters alpha for forecast model based on the historical information. For most travel data an alpha parameter is smaller than 0.40 is often effective and usually recommended. Then the best alpha has the smallest Mean Absolute Error as illustrates in the Figure 4 illustrates that the lower the value of alpha, the less responsive the forecast is to sudden change.

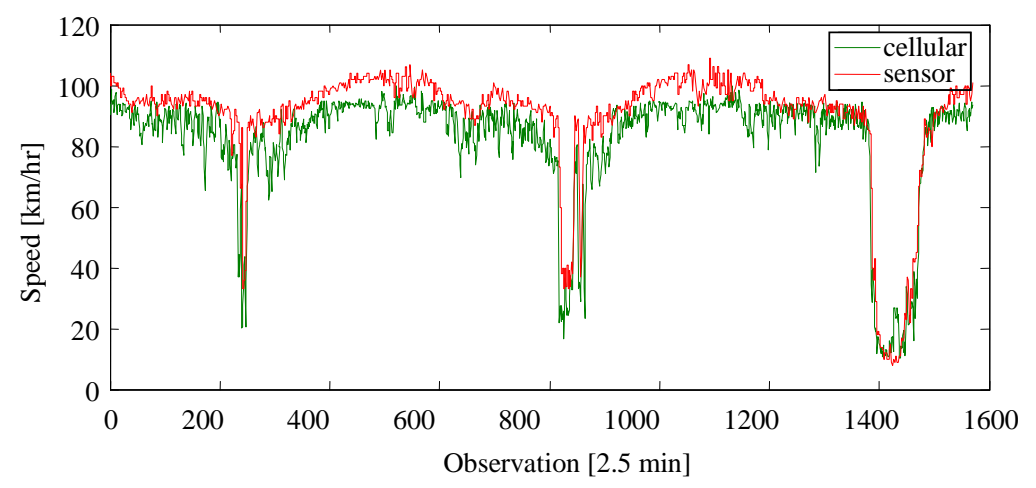

Figure 2. Cellular vs. sensor. 


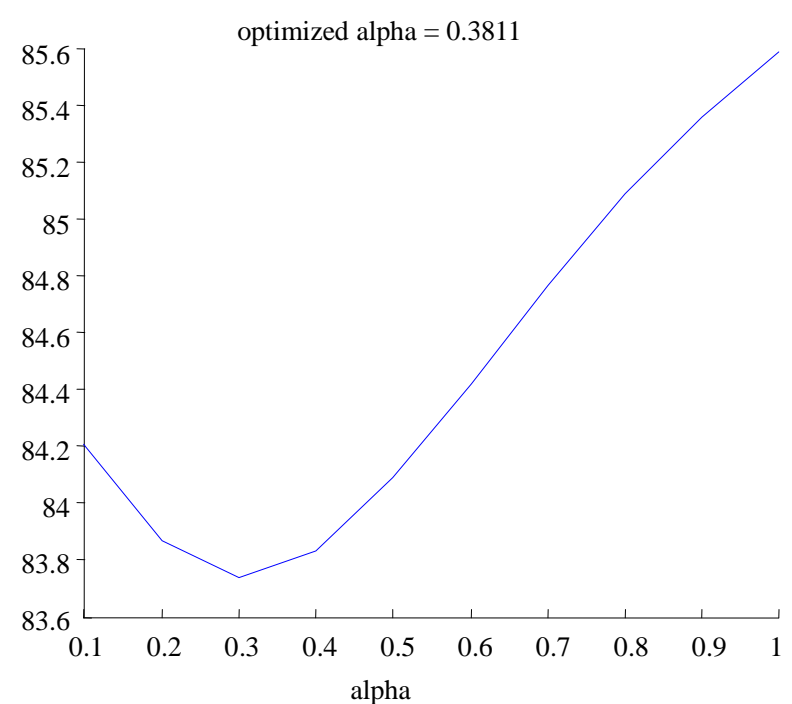

Figure 3. Smoothed parameter alpha.

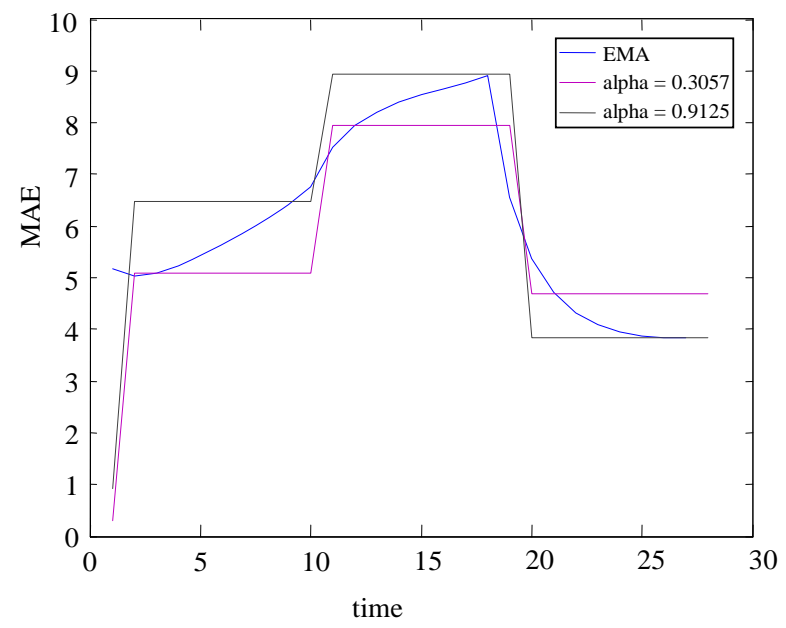

Figure 4. Mean absolute error.

\section{Simulation Results}

Various forecast schemes have been used in the cellular systems. In this section we introduce a comparison of some forecast schemes. The comparison is focused on various statistical measurements error, mean absolute error (MAE), root mean squared error (RMSE), relative absolute error (RAE), and Theil's coefficient. The statistical measurements errors are summarized in Table 1. Figure 5 illustrates the resources demand of the users in each cell related to the time interval. Hence the cognitive radio provides the needed cells with resources to avoid call blocking. Figure 6 and Figure 7 illustrate the comparison between the SMA, WMA, and EMA based on the statistical error measurements, RMSE and Theil's coefficient.
Table 1. Statistical Measurements.

\begin{tabular}{cl}
\hline MAE & $\frac{\sum_{i=1}^{n}\left|x_{i}-\bar{x}_{i}\right|}{n}$ \\
RMSE & $\sqrt{\frac{\sum_{i=1}^{n}\left(x_{i}-\bar{x}_{i}\right)^{2}}{n}}$ \\
RAE & $\frac{\frac{\sum_{i=1}^{n}\left(\left|P_{i j}-x_{i}\right|\right)}{\sum_{i=1}^{n}\left(\left|x_{i}-\bar{x}\right|\right)}}{\sqrt{\sum_{i=1}^{n}\left(x_{i}\right)^{2}}}+\sqrt{\frac{\sum_{i=1}^{n}\left(\bar{x}_{i}\right)^{2}}{n}}$ \\
\hline
\end{tabular}

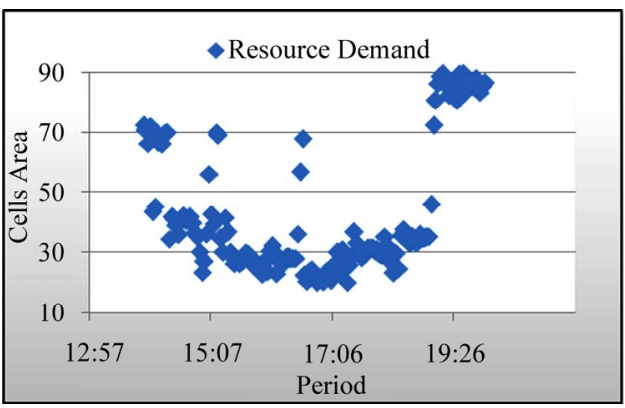

Figure 5. Resource demand.

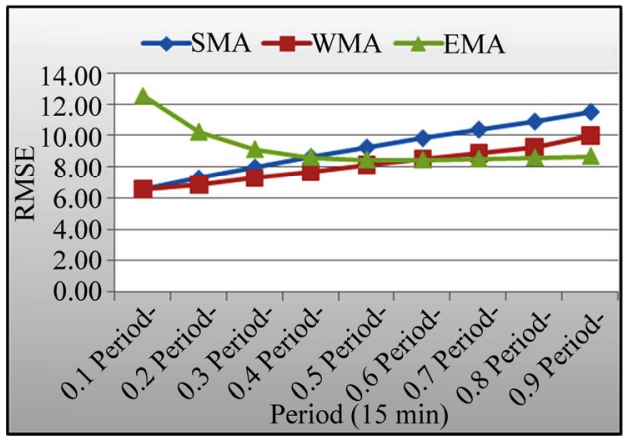

Figure 6. Moving average based RMSE.

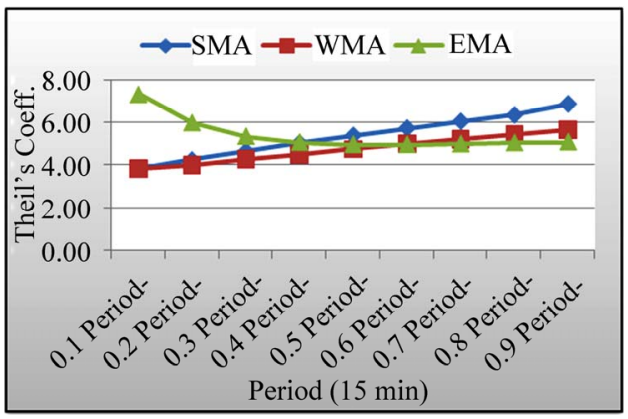

Figure 7. Moving average based Theil's coeff. 


\section{Conclusions}

In this paper we have presented a new cognitive radio sensing tools based on the short term forecasting scheme. The cognitive radio system senses its environment and acts new according to the environmental change. Furthermore we have proposed a radio cognitive based on the short-term forecast scheme to reduce the new call blocking rate and call blocking rate in handoff respectively.

\section{REFERENCES}

[1] S. K. S. Gupta and P. K. Srimani, "UpdateSearch: A New Dynamic Channel Allocation Scheme for Mobile Networks That can Adjust to System Loads," The Journal of Supercomputing, Vol. 17, No. 1, August 2000, pp. 47-65. doi:10.1023/A:1008119705225

[2] C. W. Leong, W. Zhuang, Y. Cheng and L. Wang, "Optimal Resource Allocation and Adaptive Call Admission Control for Voice/Data Integrated Cellular Networks," IEEE Transactions on Vehicular Technology, Vol. 55, No. 2, March 2006, pp. 654-669. doi:10.1109/TVT.2005.858186

[3] L. Cong, et al., "Performance Analysis of Dynamic Channel Assignment Algorithms in Cellular Mobile Systems with Hand-off," International Journal of Communication Systems, Vol. 15, No. 8, 2002, pp. 683-700. doi:10.1002/dac.558

[4] S. Anand, A. Sridharan and K. N. Sivarajan, "Performance Analysis of Channlized Cellular Systems with Dynamic Channel Allocation," IEEE Transactions on Vehicular Technology, Vol. 52, No. 4, July 2003, pp. 847859. doi:10.1109/TVT.2003.814231

[5] M. Bublin, M. Kongegger and P. Slanina, "A Cost-Function-Based Dynamic Channel Allocation and Its Limits," IEEE Transactions on Vehicular Technology, Vol. 56, No. 4, July 2007, pp. 2286-2295. doi:10.1109/TVT.2007.897657

[6] C. Politis, "Managing the Radio Spectrum," IEEE Vehicular Technology Magazine, Vol. 4, No. 1, March 2009, pp. 20-26. doi:10.1109/MVT.2008.931628

[7] M. Zafer and E. Modiano, "Blocking Probability and Channel Assignment in Wireless Networks," IEEE Transactions on Wireless Communications, Vol. 5, No. 4, April 2006, pp. 869-879. doi:10.1109/TWC.2006.1618936

[8] B. Friedlander and S. Scherzer, "Beamforming versus Transmit Diversity in the Downlink of a Cellular Communication System," IEEE Transactions on Vehicular Technology, Vol. 53, No. 4, July 2004, pp. 1023-1034. doi:10.1109/TVT.2004.830980

[9] T. Ren and R. J. La, "Downlink Beamforming Algorithms with Inter-Cell Interference in Cellular Networks," IEEE Transactions on Wireless Communications, Vol. 5, No. 10, October 2006, pp. 2814-2823.

[10] N. D. Tripathi, J. H. Reed and H. F. Vanl, "Handoff in Cellular Systems,” IEEE Personal Communications, Vol. 5, No. 6, December 1998, pp. 26-37. doi:10.1109/98.736475

[11] N. D. Tripathi, et al., "Handoff in Cellular Systems," IEEE Personal Communications, Vol. 5, No. 6, December 1998, pp. 26-37.

[12] S. Hykin, J. D. Thomson and H. J. Reed, "Spectrum Sensing for Cognitive Radio," Proceedings of the IEEE, Vol. 97, No. 5, May 2009, pp. 849-877. doi:10.1109/JPROC.2009.2015711

[13] A. F. Molisch, L. J. Greenstein and M. Shafi, "Propagation Issues for Cognitive Radio," Proceedings of the IEEE, Vol. 97, No. 5, May 2009, pp.787-804. doi:10.1109/JPROC.2009.2015704

[14] Z.-P. Li, H. Yu, Y.-C. Liu and F.-Q. Liu, "An Improved Adaptive Exponential Smoothing (IAES) Model for Short-Term Travel Time Forecasting of Urban Arterial Street,” Acta Automatica Sinica, Vol. 34, No. 11, November 2008, pp. 1404-1409.

[15] R. E. Turochy and M. Asce, "Enhancing Short-Term Traffic Forecasting with Traffic Condition Information," Journal of Transportation Engineering, Vol. 132, No. 6, June 2006, pp. 469-474.

doi:10.1061/(ASCE)0733-947X(2006)132:6(469) 